\title{
Editorial: Current Graphene Research-Going beyond the Pure Monolayer
}

The discovery of monolayer graphene, "the perfect atomic lattice," by Andre Geim and Konstantin Novoselov in 2004, opened up immense possibilities for creating and exploring new carbon-based low-dimensional materials, both for new fundamental physics and for innovative technological applications, in particular, electronics. Many of the current efforts in the broad field of graphene physics are focusing on going beyond what can be learned from and accomplished with pure monolayer graphene in a number of directions. Three papers that have been published in Physical Review X this month showcase some of these new directions.

Tying fundamental research closely to device applications, a paper by an experimental group at the University of Florida in Gainesville [Phys. Rev. X 2, 011002 (2012)], reports a comprehensive observation and understanding of a transverse Schottky barrier (or diode effect) at the interface between a monolayer graphene and a semiconductor for a surprisingly wide variety of conventional semiconductors-a development with potential applications to electronics-based devices, including chemical sensors. In the second paper [Phys. Rev. X 2, 011004 (2012)], an experimental group at the California Institute of Technology, oriented by an overarching question of whether new electronic properties will emerge from multilayer graphene, investigates trilayer graphene of a particular layer-stacking order (ABA or Bernal stacking) and finds unusual electronic transport properties, including a new sequence of quantum Hall plateaus related to lattice-symmetry breaking. And in the third [Phys. Rev. X 2, 011003 (2012)], a group of theorists from Fudan University in Shanghai, University of Science and Technology of China in Hefei, and the National Renewable Energy Laboratory in Colorado explore the avenue of chemical functionalization of pure graphene and predict that substitutional doping of pure monolayer graphene by nitrogen can lead to two new stable semiconducting compounds with properties that may make them especially useful for highperformance electronics and organic solar cells.

We are very pleased to present below brief commentaries on two of the three papers from experts in the field of graphene physics: Emanuel Tutuc from The University of Texas at Austin, and Pablo Jarillo-Herrero from the Massachusetts Institute of Technology. We hope that their insights and perspectives will both deepen and broaden your understanding and appreciation of the papers.

The Editors

Physical Review X

\section{Transport at Graphene-Semiconductor Interfaces}

Emanuel Tutuc, The University of Texas at Austin, 10100 Burnet Road, Building 160, Austin, TX 78758, USA

A Commentary on:

Rectification at Graphene-Semiconductor Interfaces: Zero-Gap Semiconductor-Based Diodes S. Tongay, M. Lemaitre, X. Miao, B. Gila, B. R. Appleton, and A. F. Hebard

Phys. Rev. X 2, 011002 (2012)

Graphene has been of significant interest as an emerging electronic material. While the majority of graphene-based electronic devices reported to date use lateral transport to benefit from the high carrier mobility in graphene, vertical transport in graphene-based heterostruc-

Published by the American Physical Society under the terms of the Creative Commons Attribution 3.0 License. Further distribution of this work must maintain attribution to the author(s) and the published article's title, journal citation, and DOI. 
tures is also under increasing scrutiny. In vertical transport devices tunneling or thermionic emission are the main transport mechanisms. In their newly published Physical Review X article, Tongay et al. provide a systematic study of transport across the interface between graphene and several common semiconductors, namely $\mathrm{Si}, \mathrm{GaAs}, \mathrm{SiC}$, and $\mathrm{GaN}$. Using current-voltage characteristics probed at different temperature and capacitance-voltage measurements, the authors determine the barrier height for electron injection by thermionic emission from the graphene layer into the semiconductor. The results are in good agreement with values estimated using the Schottky-Mott model, though subtle differences exist between electron transport across a metal-semiconductor interface and a graphene-semiconductor interface. One notable difference stems from the relatively large change in graphene work function with carrier density. In a graphene-semiconductor heterojunction this translates into a barrier height that varies depending on the applied voltage across the graphene-semiconductor interface. This study will serve as a reference for future device work using graphenesemiconductor junctions.

\section{Tunable Electronic Structure of Trilayer Graphene}

Pablo Jarillo-Herrero, Department of Physics, Massachusetts Institute of Technology, Building 13-2017, 77 Massachusetts Avenue, Cambridge, MA 02139, USA

A Commentary on: Quantum Hall Effect and Semimetallic Behavior of Dual-Gated ABA-Stacked Trilayer Graphene E. A. Henriksen, D. Nandi, and J. P. Eisenstein

Phys. Rev. X 2, 011004 (2012)

With the advent of graphene, a whole new family of novel two-dimensional electron gases (2DEGs) has become available to condensed matter physicists. Besides the familiar massless Dirac fermions that emerge in monolayer graphene, several other types of electronic behaviors are possible by simply stacking graphene sheets on top of each other. In the case of Bernalstacked or ABA trilayer graphene, the electronic structure is approximately described by monolayer-like and bilayer-like subbands at low energy. The interplay between these two subbands at high magnetic field gives rise to an intriguing quantum Hall response different from that of the more familiar monolayer and bilayer graphene cases. The very few studies of quantum Hall effect in ABA trilayer graphene so far have been limited to single back-gated devices, and hence the effects of a symmetry-breaking transverse electric displacement field on the electronic structure and quantum Hall effect have not been studied experimentally. In their interesting paper published in Physical Review X, Henriksen, Nandi, and Eisenstein have investigated ABA dual-gated trilayer graphene both at zero magnetic field and in the quantum Hall regime. This dual-gate geometry allows them to independently control the charge density and transverse displacement field. In the quantum Hall regime, the observed electron-hole asymmetry supports a semimetallic band overlap picture, in agreement with calculations taking into account a more complete tight-binding parametrization of trilayer graphene. In addition to that, the ability to control the displacement field enables them to introduce a potential difference between the layers and break the mirror symmetry in ABA trilayer graphene resulting in Landau level splittings. At zero magnetic field, they observe that the resistivity at the charge neutrality point increases with increasing displacement field (for low fields), similar to bilayer graphene, but in stark contrast to previously reported results. This work motivates further studies on locally gated lateral heterostructures in trilayer graphene, such as $p-n$ junctions and Fabry-Perot interferometers, where the unique properties of this novel twodimensional electron gas can be explored. 
About the two commentary authors:

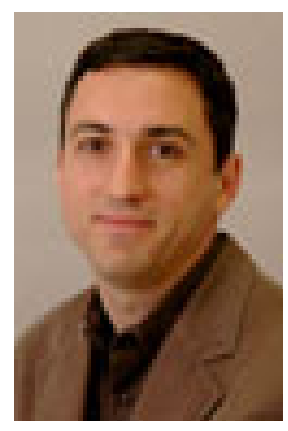

Emanuel Tutuc is Assistant Professor at The University of Texas at Austin, within the Department of Electrical and Computer Engineering. His group carries out experimental research with an emphasis on electron transport in graphene and semiconductor nanowires.

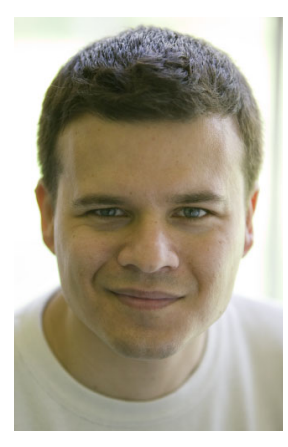

Pablo Jarillo-Herrero is Assistant Professor of Physics at the Massachusetts Institute of Technology. His research interests span quantum transport and optoelectronics in low dimensional materials, such as graphene and topological insulators.

Published 31 January 2012

DOI: 10.1103/PhysRevX.2.010001 